ESAIM: PROCEEDINGS, September 2007, Vol.19, 121-125

Christophe Andrieu \& Dan Crisan, Editors

DOI: $10.1051 /$ proc:071916

\title{
NUMERICAL SOLUTIONS FOR A CLASS OF SPDES OVER BOUNDED DOMAINS $*, * *$
}

\author{
DAN CRISAN ${ }^{1}$ AND JIE XIONG ${ }^{2}$
}

\begin{abstract}
The optimal filter for a bounded signal with reflecting boundary is approximated by the (un-weighted) empirical measure of a finite interacting particle system. The main motivation of this un-weighted empirical measure representation is to overcome the slow convergence rate of the weighted one because of the exponential growth of the variance of individual weight of the particle. The finite system of SDEs with reflecting boundary is then solved numerically by Euler scheme.
\end{abstract}

\section{INTRODUCTION}

In Crisan and Xiong [8], we studied a McKean-Vlasov representation for the Kushner-FKK equation arising from the nonlinear filtering theory. The motivation of that paper is to search for effective numerical method to solve the filtering equation. The present paper is a follow up of that effort. More specifically, we shall present an approximation of the optimal filter by the empirical (un-weighted) measure of an interacting finite particle system.

Numerical solutions to the filtering equation has attracted intensive research effort recently. One of the methods is to use the interacting particle system approximation. A direct Monte-Carlo approximation is studied by Kurtz and Xiong ([12], [13], [14]) as a special case of their study of a large class of SPDEs. The drawback of that method is that the variance of the weight grows exponentially fast in time and, so does the approximation error. In a series of papers ([1], [2], [3], [4], [5], [6], [7], [9], [10], [11]), many authors studied the approximation using branching interacting particle systems (BIPS). Comparing with the direct Monte-Carlo approximation, the BIPS method update the information at each time step, i.e., to drop particles with small mass. This overcomes the drawback of the Monte-Carlo method. However, because of the variance of the weight, the convergence rate of the BIPS is still not satisfactory from a practical point of view. To overcome this, we initiated in [8] the study of the representation of the optimal filter by un-weighted McKean-Vlasov equation in hoping to obtain an approximation by un-weighted empirical measure of a finite particle system. However, the condition (ES) imposed in [8] is hard to verify.

In this paper, we use an additional approximation. Namely, we approximate the diffusion process in $\mathbb{R}^{d}$ by a diffusion $X_{t}$ on $D=\left\{x \in \mathbb{R}^{d}:|x| \leq R\right\}$ with reflecting boundary. Let $\sigma: \mathbb{R}^{d} \rightarrow \mathbb{R}^{d \times d}$ and $b: \mathbb{R}^{d} \rightarrow \mathbb{R}^{d}$ be bounded and Lipschitz continuous maps and $a=\sigma \sigma^{*}$, here $\sigma^{*}$ denotes the transpose of the matrix $\sigma$. We

\footnotetext{
* Research of J. X. is supported partially by NSA

** This work was initiated when the second author visits Imperial College. The hospitality of Imperial College and financial support from London Mathematical Society are gratefully acknowledged.

1 Department of Mathematics, Imperial College London, 180 Queen's Gate, London SW7 2BZ, UK.

2 Department of Mathematics, University of Tennessee, Knoxville, TN 37996-1300, USA; and, Department of Mathematics, Hebei Normal University, Shijiazhuang 050016, PRC
} 
consider the filtering problem with the signal $X_{t}$ and the observation process

$$
Y_{t}=\int_{0}^{t} h\left(X_{s}\right) d s+W_{t}
$$

where $h: \mathbb{R}^{d} \rightarrow \mathbb{R}^{m}$ is a continuous function. Then the optimal filter $\pi_{t}(\cdot) \equiv P\left(X_{t} \in \cdot \mid \mathcal{F}_{t}^{Y}\right)$ is the unique solution to the following Kushner-FKK equation:

$$
d\left\langle\pi_{t}, f\right\rangle=\left\langle\pi_{t}, L f\right\rangle d t+\left\langle\pi_{t},\left(h-\left\langle\pi_{t}, h\right\rangle\right) f\right\rangle d \nu_{t}
$$

where

$$
L=b^{*} \nabla+\frac{1}{2} \sum_{j, k=1}^{d} a^{j k} \partial_{j k}^{2}, \quad D(L)=\left\{f:\left.x^{*} \nabla f\right|_{\partial D}=0\right\}
$$

is the generator of $X_{t}$ and $\nu_{t}$ is an $m$-dimensional $\mathcal{F}_{t}^{Y}$-adapted Brownian motion, called the innovation process. The aim of this paper is to study the numerical solution of $\pi_{t}$. As being indicated in [8], we can approximate the Brownian motion $\nu_{t}$ by a smooth process $\tilde{\nu}_{t}$ and, by robustness, the optimal filter $\pi_{t}$ can be approximated by the solution to the following PDE:

$$
\frac{d}{d t}\left\langle\mathcal{I}_{t}, f\right\rangle=\left\langle\mathcal{I}_{t}, L f\right\rangle+\left\langle\mathcal{I}_{t}, \alpha_{t}^{\mathcal{I}_{t}} f\right\rangle
$$

where $\alpha_{t}=h \frac{d}{d t} \tilde{\nu}_{t}$ is a bounded smooth function and

$$
\alpha^{\mathcal{I}}=\alpha-\langle\mathcal{I}, \alpha\rangle .
$$

In this paper, we will give a numerical scheme in approximating $\mathcal{I}_{t}$.

The following conditions will be assumed throughout this article.

(B) There exists a constant $K$ such that for any $t \in[0, T]$ and $x \in D$, we have

$$
|b(x)|+|\sigma(x)|+\left|\alpha_{t}(x)\right| \leq K .
$$

(Lip) For any $t \in[0, T]$ and $x, y \in D$, we have

$$
|b(x)-b(y)|+|\sigma(x)-\sigma(y)|+\left|\alpha_{t}(x)-\alpha_{t}(y)\right| \leq K|x-y| .
$$

(UE) There exists a constant $K_{0}>0$ such that for any $x \in D$, the matrix $a(x)-K_{0} I$ is non-negative definite.

This article is organized as follows: In section 1, we give a particle representation of $\mathcal{I}_{t}$. Based on this representation, we approximate $\mathcal{I}_{t}$ by a finite particle system in Section 2. Finally, in Section 3, we obtain a numerical solution for this system of finite many SDEs with reflecting boundary conditions by making use of the results of Petterson [15] (see also Slominski [17]).

\section{Particle system Representation}

Consider an infinite system of interacting particles governed by the following stochastic differential equations (SDEs):

$$
d X_{t}^{i}=\tilde{b}_{t}\left(\mathcal{I}_{t}, X_{t}^{i}\right) d t+\sigma\left(X_{t}^{i}\right) d B_{t}^{i}+\mathcal{N}\left(X_{t}^{i}\right) d K_{t}^{i}, \quad i=1,2, \cdots
$$

and

$$
\mathcal{I}_{t}=\lim _{n \rightarrow \infty} \frac{1}{n} \sum_{i=1}^{n} \delta_{X_{t}^{i}}
$$


where $K_{t}^{i}$ is the local time of $X_{t}^{i}$ at the boundary of $D, \mathcal{N}(x)$ is the unit normal vector of $\partial D$ at $x \in \partial D$,

$$
\tilde{b}_{t}\left(\mathcal{I}_{t}, x\right)=b(x)+\frac{\Lambda_{\mathcal{I}_{t}} \alpha_{t}(x)}{\mathcal{I}_{t}(x)}
$$

and

$$
\Lambda_{\mathcal{I}} \alpha(x)=\frac{1}{\omega_{d}} \int_{\mathbb{R}^{d}} \frac{(y-x) \alpha^{\mathcal{I}}(y)}{\|y-x\|^{d}} \mathcal{I}(d y)
$$

$\omega_{d}$ is the surface area of the $d$-dimensional unit sphere $S_{d-1}$. This makes $X_{t}^{i}$ a diffusion on $D$ with reflecting boundary.

To derive the equation satisfied by $\mathcal{I}_{t}$, we need the following lemma.

Lemma 1.1. If $y \neq 0$, then

$$
\int_{D} \frac{\nabla^{*} f(x)(y-x)}{\|y-x\|^{d}} d x=-\int_{\partial D} f(x) d S+\omega_{d} f(y) .
$$

Based on this lemma, we can derive the equation satisfied by $\mathcal{I}_{t}$.

Theorem 1.2. $\mathcal{I}_{t}$ satisfies the following equation

$$
\frac{d}{d t}\left\langle\mathcal{I}_{t}, f\right\rangle=\left\langle\mathcal{I}_{t}, L f\right\rangle+\left\langle\mathcal{I}_{t}, \alpha_{t}^{\mathcal{I}_{t}}\right\rangle
$$

Next theorem gives the lower bound of $\mathcal{I}_{t}$ which will be useful in next section.

Theorem 1.3. Suppose $\alpha$ is bounded and $\mathcal{I}_{0}(u) \geq \epsilon_{1}>0$. Then, there exists a constant $c>0$ such that $\mathcal{I}_{t}(x) \geq c$ for all $t$ and $x$.

\section{Finite PARTiCle SYSTEM APPROXimATiON}

In this section, we consider the approximation to the solution to (1.3) based on the particle system representation (1.1-1.2).

We fix $\epsilon, \delta>0$ and consider the following finite system: For $i=1,2, \cdots n$,

$$
d X_{t}^{n, \epsilon, \delta, i}=\tilde{b}_{t}^{\epsilon, \delta}\left(\mathcal{I}_{t}^{n, \epsilon, \delta}, X_{t}^{n, \epsilon, \delta, i}\right) d t+\sigma\left(X_{t}^{n, \epsilon, \delta, i}\right) d B_{t}^{i}+\mathcal{N}\left(X_{t}^{n, \epsilon, \delta, i}\right) d K_{t}^{i}
$$

and

$$
\mathcal{I}_{t}^{n, \epsilon, \delta}=\frac{1}{n} \sum_{i=1}^{n} \delta_{X_{t}^{n, \epsilon, \delta, i}}, \quad \tilde{b}_{t}^{\epsilon, \delta}(\mu, x)=b(x)+\frac{\Lambda_{\mu} \alpha_{t}(x)}{T_{\epsilon} \mu(x)+\delta}
$$

where for any finite measure $\mu$,

$$
T_{\epsilon} \mu(x)=(2 \pi \epsilon)^{-\frac{d}{2}} \int \exp \left(-\frac{|x-y|^{2}}{2 \epsilon}\right) \mu(d y) .
$$

Next three theorems give the limit of $\mathcal{I}_{t}^{n, \epsilon, \delta}$ as $n \rightarrow \infty, \epsilon \rightarrow 0$ and $\delta \rightarrow 0$ respectively.

Theorem 2.1. For $\epsilon, \delta>0$ fixed, we have

$$
\lim _{n \rightarrow \infty} \mathcal{I}_{t}^{n, \epsilon, \delta}=\mathcal{I}_{t}^{\epsilon, \delta}
$$


which is the unique solution to

$$
\frac{d}{d t}\left\langle\mathcal{I}_{t}^{\epsilon, \delta}, f\right\rangle=\left\langle\mathcal{I}_{t}^{\epsilon, \delta}, L f\right\rangle+\left\langle\mathcal{I}_{t}^{\epsilon, \delta}, \nabla^{*} f \frac{\Lambda_{\mathcal{I}_{t}^{\epsilon, \delta}} \alpha_{t}}{T_{\epsilon} \mathcal{I}_{t}^{\epsilon, \delta}+\delta}\right\rangle .
$$

Next, we consider the convergence of $\mathcal{I}_{t}^{\epsilon, \delta}$ as $\epsilon \rightarrow 0$ and $\delta$ being fixed. To this end, we need some estimates

Lemma 2.2. There exists a constant $C(\delta, T)$ such that

$$
\sup _{x \in D} \mathcal{I}_{t}^{\epsilon, \delta}(x) \leq C(\delta, T)
$$

Lemma 2.3. There exists a constant $C(\delta, T)$ such that

$$
\sup _{x \in D}\left|\nabla \mathcal{I}_{t}^{\epsilon, \delta}(x)\right| \leq C(\delta, T)
$$

As a consequence of Lemmas 2.2 and 2.3, we have

Theorem 2.4. For $\delta>0$ fixed, we have

$$
\lim _{\epsilon \rightarrow 0} \mathcal{I}_{t}^{\epsilon, \delta}=\mathcal{I}_{t}^{\delta}
$$

which is the unique solution to

$$
\frac{d}{d t}\left\langle\mathcal{I}_{t}^{\delta}, f\right\rangle=\left\langle\mathcal{I}_{t}^{\delta}, L f\right\rangle+\left\langle\mathcal{I}_{t}^{\delta}, \nabla^{*} f \frac{\Lambda_{\mathcal{I}_{t}^{\delta}} \alpha_{t}}{\mathcal{I}_{t}^{\delta}+\delta}\right\rangle
$$

Finally, we take the last limiting procedure of this section.

\section{Theorem 2.5.}

$$
\lim _{\delta \rightarrow 0} \mathcal{I}_{t}^{\delta}=\mathcal{I}_{t}
$$

\section{NumericAl SOLUTION}

In this section we use Euler scheme to solve the SDE system (2.1). For simplicity of notation, we drop the superscripts $n, \epsilon, \delta$ in this section. The system (2.1) becomes

$$
\left\{\begin{array}{l}
d X_{t}^{i}=\tilde{b}_{t}\left(\mathcal{I}_{t}, X_{t}^{i}\right) d t+\sigma\left(X_{t}^{i}\right) d B_{t}^{i}+\mathcal{N}\left(X_{t}^{i}\right) d K_{t}^{i} \\
\mathcal{I}_{t}=\frac{1}{n} \sum_{i=1}^{n} \delta_{X_{t}^{i}}, \quad \tilde{b}_{t}(\mu, x)=b(x)+\frac{\Lambda_{\mu} \alpha_{t}(x)}{T_{\epsilon} \mu(x)+\delta}
\end{array}\right.
$$

Let $0=t_{0}<t_{1}<\cdots<t_{c_{\gamma}}+T$ be a partition of $[0, T]$ with mesh size $\gamma=\max _{1 \leq k \leq c_{\gamma}} \Delta t_{t}$ where $\Delta t_{k}=t_{k}-t_{k-1}$. Let $\Pi_{D}$ be the projection to $D$ (i.e. $\Pi_{D} x$ is the point in $D$ which is the closest to $x$ ). For $0 \leq t<t_{1}$, define $X_{t}^{\gamma, i}=x_{0}^{i}$; and for $t_{k} \leq t<t_{k+1}, k \geq 1$,

$$
\left\{\begin{array}{l}
X_{t}^{\gamma, i}=\Pi_{D}\left\{X_{t_{k-1}}^{\gamma, i}+\tilde{b}_{t_{k-1}}\left(\mathcal{I}_{t_{k-1}}^{\gamma}, X_{t_{k-1}}^{\gamma, i}\right) \Delta t_{k}+\sigma\left(X_{t_{k-1}}^{\gamma, i}\right) \Delta B_{t_{k}}^{i}\right\} \\
\mathcal{I}_{t}^{\gamma}=\frac{1}{n} \sum_{i=1}^{n} \delta_{X_{t}^{\gamma, i}}^{\gamma,}
\end{array}\right.
$$

where $\Delta B_{t_{k}}^{i}=B_{t_{k}}^{i}-B_{t_{k-1}}^{i}$.

Theorem 3.1. There exists a constant $C_{\epsilon, \delta}$ such that

$$
\sup _{0 \leq t \leq T} \mathbb{E} \rho\left(\mathcal{I}_{t}^{n, \epsilon, \delta, \gamma}, \mathcal{I}_{t}^{n, \epsilon, \delta}\right)^{2} \leq C_{\epsilon, \delta}\left(\gamma \log \frac{1}{\gamma}\right)^{1 / 2} .
$$


Combining (3.1), (2.3), (2.6) and (2.8) we see that as $n \rightarrow \infty$ and $\epsilon, \delta, \gamma \rightarrow 0, \mathcal{I}_{t}^{n, \epsilon, \delta, \gamma}$ converges to $\mathcal{I}_{t}$ which is the unique solution to the equation $(1.3)$.

\section{REFERENCES}

[1] D. CRISAN, Numerical methods for solving the stochastic filtering problem, Numerical methods and stochastics (Toronto, ON, 1999), 1-20, Fields Inst. Commun., 34, Amer. Math. Soc., Providence, RI, 2002.

[2] D. Crisan, J. Gaines, T. Lyons, Convergence of a branching particle method to the solution of the Zakai equation. SIAM J. Appl. Math. 58 (1998), no. 5, 1568-1590 (electronic).

[3] D. Crisan, T. Lyons, A particle approximation of the solution of the Kushner-Stratonovitch equation Probab. Theory Related Fields 115 (1999), no. 4, 549-578.

[4] D. Crisan, P. Del Moral T. Lyons, Interacting particle systems approximations of the Kushner-Stratonovitch equation. Adv. in Appl. Probab. 31 (1999), no. 3, 819-838.

[5] D. Crisan, Particle filters - a theoretical perspective. Sequential Monte Carlo methods in practice, 17-41, Stat. Eng. Inf. Sci., Springer, New York, 2001.

[6] D. CRisan, A. Doucet, A survey of convergence results on particle filtering methods for practitioners. IEEE Trans. Signal Process. 50, no. 3, pp 736-746, 2002.

[7] D. CRISAN, Exact rates of convergence for a branching particle approximation to the solution of the Zakai equation Ann. Probab. 31 (2003), no. 2, 693-718.

[8] D. Crisan and J. Xiong. Approximate McKean-Vlasov Representations for a class of SPDEs. In this volume.

[9] P. Del Moral (2004) Feynman-Kac formulae. Genealogical and interacting particle systems with applications. Probability and its Applications, Springer-Verlag, New York.

[10] P. Del Moral, A. Guionnet (1999), Central limit theorem for nonlinear filtering and interacting particle systems, Ann. Appl. Probab. 9, no. 2, 275-297.

[11] P. Del Moral, L. Miclo (2000), Branching and interacting particle systems approximations of Feynman-Kac formulae with applications to non-linear filtering. Séminaire de Probabilités, XXXIV, 1-145, Lecture Notes in Math., 1729, Springer, Berlin.

[12] T. Kurtz and J. Xiong (1999). Particle representations for a class of nonlinear SPDEs. Stochastic Processes and their Applications 83, 103-126.

[13] T. Kurtz and J. Xiong (2000). Numerical solutions for a class of SPDEs with application to filtering. Stochastics in Finite and Infinite Dimension: In Honor of Gopinath Kallianpur. Edited by T. Hida, R. Karandikar, H. Kunita, B. Rajput, S. Watanabe and J. Xiong. Trends in Mathematics. Birkhauser.

[14] T. Kurtz and J. Xiong (2004). A stochastic evolution equation arising from the fluctuation of a class of interacting particle systems. Communication Mathematical Sciences Vol. 2, 325-358.

[15] R. Pettersson (1997). Penalization schemes for reflecting stochastic differential equations. Bernoulli 3, No. 4, $403-414$.

[16] L. Saloff-Coste (2002). Aspects of Sobolev-Type Inequalities. Cambridge University Press.

[17] L. Slominski (2001). Euler's approximations of solutions of SDEs with reflecting boundary. Stochastic Processes and their Applications 94, No. 2, 317-337. 